\title{
COLLECTIVITY, CHAOS, AND COMPUTERS
}

\author{
CALVIN W. JOHNSON \\ Department of Physics, San Diego State University \\ 5500 Campanile Drive \\ San Diego, CA 92182-1233, USA \\ E-mail: johnson@physics.sdsu.edu
}

\begin{abstract}
Two important pieces of nuclear structure are many-body collective deformations and single-particle spin-orbit splitting. The former can be well-described microscopically by simple $\mathrm{SU}(3)$ irreps, but the latter mixes $\mathrm{SU}(3)$ irreps, which presents a challenge for large-scale, ab initio calculations on fast modern computers. Nonetheless, SU(3)-like phenomenology remains even in the face of strong mixing. The robustness of band structure is reminiscent of robust, pairing collectivity that arises from random two-body interactions.
\end{abstract}

\section{Nuclear epistemology}

The goal of nuclear structure theory is to understand experimental spectra, but what do we mean by understand?

There are two routes to understanding. One is the brute force, extreme reductionism of $a b$ initio computations ${ }^{1}$ : one starts from bare nucleonnucleon scattering data and eventually computes many-body binding energies, spectra, transition rates, etc. This is of course very appealing to physicists, and I believe such ab initio calculations are among the most important nuclear structure results of the past decade, but it has the very obvious danger of getting lost in the numerical details. Furthermore, bruteforce calculations are limited by available computing power.

The alternate is back-of-the-envelope reasoning: simple, primarily analytic models that are easy to intuit. Algebraic models are prime examples $^{2,3}$. The danger here is that the simple picture may not accurately represent the microscopic physics.

Ideally, one would like to combine these two: perform microscopically detailed $a b$ initio calculations built upon basis state constructed from algebraic models. Such a hybrid approach would certainly allow one greater insight into the microscopic calculation, and would, one hopes, be much 
more efficient.

More specifically, I wish to address the possibility of using SU(3) irreps as a best basis for large-scale microscopic calculations. The biggest obstacle is spin-orbit splitting that arises from the nuclear mean field.

\section{A brief guide to nuclear structure}

Nuclear structure is driven by several competing degrees of freedom. First, the nucleus has a mean field, which allowed Haxel, Jensen, and Suess, and Mayer ${ }^{4}$ to propose the non-interacting shell model. One of the primary features of the nuclear mean field is a strong spin-orbit splitting. Spin-orbit splitting arises naturally from a non-relativistic reduction of the Dirac equation; as the nucleus is more relativistic than the atom, it is understandable that spin-orbit splitting is very small in atomic physics but is a large feature in nuclear physics. (In fact it becomes so large that it gives rise to pseudospin; see ref. ${ }^{5,6}$ and the contributions by Ginocchio and van Isacker in this volume.) Eventually from the noninteracting shell model developed the interacting shell model, where one chooses a finite set of fermion shell model states and diagonalizes a Hamiltonian in that space.

The starting point of the shell model is the independent particle assumption: the component protons and neutrons interact primarily with the mean field. This is a simplification: there are correlations between the nucleons, and most important are the collective correlations. One well known form of collectivity is pairing, whereby fermions of opposite (angular) momentum couple to zero. This is a pervasive feature of cold, dense fermion systems, but it play only a peripheral role in this paper. Through the seniority model $^{2}$ one can understand pairing in terms of microscopic fermion states.

Instead the collectivity that I will pay most attention to, and the one which has been the primary focus of Jerry Draayer's work, is quadrupole deformation. One can have both quadrupole vibrations and "static" quadrupole deformations that lead to rotational bands. Quadrupole deformations arise naturally out of the semiclassical liquid drop model of the nucleus, and can be treated more formally in the Bohr-Mottelson model and its generalization to geometric-collective models ${ }^{7}$.

One of the great breakthroughs in nuclear stucture physics was discovering how to connect collective motion to the underlying fermion microphysics. Elliot's SU(3) model ${ }^{8}$ and its successors showed how one could map rotational motion easily onto the fermion shell model. Furthermore, 
as Rowe ${ }^{3}$ has emphasized, SU(3) maps also onto the Bohr-Mottelson and similar models, thus providing a critical bridge between macroscopic and microscopic pictures. SU(3), at least as phenomenology, describes beautifully many features of nuclear spectra. But how well do microscopic SU(3) wavefunctions match 'realistic' microscopic wavefunctions? That is a question I will return to.

One of the most powerful tools for nuclear structure is the spherical interacting shell model. Here one starts by assuming a spherically symmetric mean field, so that all single-particle states have good $j$. The model space is partitioned into subspaces by single-particle configurations: one subspace might be, for example, all states with the configuration $\left(0 d_{5 / 2}\right)^{3}\left(1 s_{1 / 2}\right)^{1}\left(0 d_{3 / 2}\right)^{2}$. In fact, because the Hamiltonian is rotationally invariant, one can restrict to the states with a fixed total $M$ (that is, $J_{z}$ ) and hence programs that work in this basis are often referred to as $M$-scheme codes. The many-body Hamiltonian matrix elements are then computed in this basis.

Because the total angular momentum operator $J^{2}$ does not connect across configurations, it is easy to construct a many-body model space for which angular momentum is a good quantum number. Futhermore, spinorbit splitting can be treated nearly trivially in such model spaces. What cannot be treated easily is deformation: deformation mixes many configurations, and typically one needs to add effective charges to get correct magnitudes for E2 transitions, etc.

Despite this important drawback, $M$-scheme and related codes are very popular today. Some of the older codes, such as the Glasgow code ${ }^{9}$ or $\mathrm{OXBASH}^{10}$, store the many-body Hamiltonian on disk. This works for a basis size of up to about half a million basis states. Beyond that, more recent shell-model codes such as ANTOINE ${ }^{11}$ or REDSTICK ${ }^{12}$ recompute the Hamiltonian many-body matrix elements on-the-fly. With hard work and clever coding, this can be very efficient.

The interacting shell model in a spherical basis is not the only possible approach. One of Jerry Draayer's great achievements has been to construct, with a series of collaborators, $\mathrm{SU}(3)$ shell model codes ${ }^{13,14,15,16,17}$. At the heart of these codes are Slater determinants in a cylindrical rather than a spherical single-particle basis. This allows one rather easily to get fermion representations of $\mathrm{SU}(3)$.

A useful generalization of the $\mathrm{SU}(3)$ model is the symplectic model ${ }^{18}$, which unifies quadrupole operators with center-of-mass motion. This allows one to treat multi- $\hbar \Omega$ shell spaces and to project out exactly spurious center- 
of-mass motion. Futhermore one can generalize the SU(3) technology to symplectic calculations ${ }^{19}$.

Other properties, however, are not as easy. Projection of good angular momentum is not as straightforward as for spherical shell-model configurations. (This can probably be made more efficient.)

Unfortunately one remaining feature of nuclear structure remains a potential obstacle: spin-orbit splitting from the mean field, which I take up in the next section.

\section{Mixed or pure SU(3)-that is the question}

A number of well-known phenomenological interactions mix SU(3) irreps. One is pairing ${ }^{16}$. More germane to my discussion is single-particle spinorbit splitting which arises from the nuclear mean-field ${ }^{17,20}$. The bottom line: in calculations in the $s d$ - and lower $p f$-shells one finds that singleparticle spin-orbit splitting is by far the most important source of mixing of $\mathrm{SU}(3)$ irreps. If one eliminates spin-orbit splitting, then mixing of $\mathrm{SU}(3)$ irreps is enormously reduced ${ }^{20,21}$.

We investigated the role of spin-orbit splitting as follows ${ }^{20}$. First, we took 'realistic' interactions: Wildenthal's USD interaction in the $s d$-shell ${ }^{22}$ and the monopole-modified KB3 interaction in the $p f$-shell ${ }^{23}$. These interactions started life as exact $G$-matrix effective interactions reduced from nucleon-nucleon forces, with some empirical adjustments fit to hundreds of levels and decays. These interactions are by no means schematic and were derived blindly with respect to $\mathrm{SU}(3)$.

We computed for various nuclides the 'exact' wavefunctions for these complicated, messy interactions. Using a Lanczos moment method, similar to that developed to compute Gamow-Teller strength distributions ${ }^{24}$, we were able to compute the distribution of the exact wavefunction onto $\mathrm{SU}(3)$ irreps, without having to compute all the $\mathrm{SU}(3)$ eigenstates.

In the $s d$-shell and particularly in the $p f$-shell we found the wavefunctions to be fragmented over many $\mathrm{SU}(3)$ irreps. When we simply eliminated the spin-orbit splitting, and nothing else, then the fragmentation was enormously reduced, even for $p f$-shell nuclides. One can fairly interpret the fragmentation of $\mathrm{SU}(3)$ irreps due to realistic spin-orbit splitting to mean that wavefunctions of pure or nearly pure $\mathrm{SU}(3)$ irreps are not very realistic on a microscopic level.

Despite this fragmentation, the energy of deformation is larger than spin-orbit splitting. If one considers, for example, ${ }^{24} \mathrm{Mg}$ in the $s d$-shell, the 
leading $(8,4)$ irrep of $\mathrm{SU}(3)$ outperforms the simplest spherical configuration $\left(d_{5 / 2}\right)^{8}$, in terms of binding energy, B(E2) values, etc. ${ }^{25}$.

While that is impressive, another calculation using a single Hartree-Fock state projected onto good angular momentum, showed that for a number of $s d$ - and lower $p f$-shell nuclides the projected HF state outperforms a single $\mathrm{SU}(3)$ irrep $^{21}$. Again the difference is driven almost entirely by the single-particle spin-orbit splitting: if one removes spin-orbit splitting, the difference between a projected HF state and the leading irrep is small.

So clearly one needs both deformation and spin-orbit splitting. Any route that neglects one over the other has to work hard to catch up. But which route? A shell-modeler faces a large number of choices for basis states: spherical shell model configuations; SU(3) irreps; configurations built upon deformed Hartree-Fock ${ }^{26}$; or a 'mixed-mode' basis ${ }^{25}$ combining two or more of these. Which is best, and how can we tell which is 'best'?

As discussed above, an $\mathrm{SU}(3)$ basis can be very illuminating in terms of the physics. For very-large-scale calculations, however, one must be concerned with computational effciency. For example, in full $0 \hbar \Omega$ shellmodel calculations, codes working in a spherical basis are still much more efficient than an $\mathrm{SU}(3)$ basis; the former can compute ${ }^{24} \mathrm{Mg}$ roughly ten times faster than the latter. Let me emphasize that is for the full space including all configurations; the motivation of using an $\mathrm{SU}(3)$ basis is the belief that one can truncate drastically to a smaller and more efficient basis and still get a very good description of the spectrum and wavefunctions.

Unfortunately $\mathrm{SU}(3)$ isn't always as efficient as one would hope, due to mixing of irreps due to spin-orbit splitting (much as quadrupole deformation strongly mixes configurations in the spherical shell model). In a hybrid approach, Gueorgueiv et $\mathrm{al}^{25}$ showed that an oblique-that is, nonorthogonalbasis consisting of a few $\mathrm{SU}(3)$ irreps and a few spherical configurations could work very well, requiring only a few states: the SU(3) irreps encoded deformation and the spherical configurations encoded spin-orbit splitting.

While this sounds marvelous, the problem with such a statement is that those few states are not easy to represent in the computer. Therefore, in order to truly diagnose how efficient a basis is, I make the following observation and proposal. Most modern, large-scale, interacting shell-model codes use Slater determinants as the fundamental internal representation. The Slater determinant is in a single-particle basis: spherical, cylindrical, or other such as Hartree-Fock. We can discuss usefully the computational efficiency in terms of the number of Slater determinants needed to represent a state or to project out a good quantum number. 
(Let me also note that there is a significant difference between a computationally efficient basis and one that illuminates the physics. A handful of states, while computationally inefficient, can still shed significant light upon the general structure of the state. But it is also important, when proposing a basis, to distinguish between computational efficiency and "physics efficiency.")

One can project out states of good angular momentum in a spherical shell-model space relatively efficiently, requiring only a few tens or a few hundred Slater determinants, and all within a single configuration. Spinorbit splitting is trivial; and if one crosses major harmonic-oscillator shells, it is possible to project out spurious center-of-mass motion as well, as long as one includes the right set of configurations. On the other hand, building in deformation requires many configurations.

In the cylindrical shell model, one can get the leading SU(3) irreps, and thus deformation, easily with just a few Slater determinants. Furthermore, if one has multi-shell calculations and uses the symplectic extension, it is possible to project out spurious center-of-masss motion. On the other hand, as presently written in the $\mathrm{SU}(3)$ shell-model codes, projection of good angular momentum is not very efficient, requiring several hundred or even more than a thousand Slater determinants. (Indeed, this is why, for exactly the same model space, spherical shell-model codes are faster than SU(3) codes; the latter can probably be sped up.) Finally, spin-orbit splitting can only be handled by mixing many irreps.

What about Hartree-Fock based states? They include deformation and spin-orbit splitting, and the amount of effort needed to project out good angular momentum is roughly comparable to $\mathrm{SU}(3)$ irreps. Unfortunately, I believe that consistent projection of spurious center-of-mass motion could be problematic (for reasons I do not have space to discuss here); and this is critical for large-basis calculations.

Ideally one would like to combine $a b$ initio calculations with the lessons learned from algebraic models. The latter describe deformation, and in the symplectic extension can project out spurious center-of-mass motion, but are strongly mixed by spin-orbit splitting. I think some generalized approach is needed, such as the "optimal basis states" 27 which combine symplectic states with generator coordinate methods. Such a proposal seems very appealing, but needs further study. 


\section{Persistence of collectivity}

Despite mixing of SU(3) irreps by spin-orbit splitting, the resulting spectra -not only energy levels but also B(E2) ratios-can be described very well phenomenologically by $\mathrm{SU}(3)$. That is to say, if one numerically solves a Hamiltonian of the form $Q \cdot Q+l \cdot s$, the resulting wavefunctions will strongly mix SU(3) irreps, but one can fit the energy levels and B(E2)s to analytic $\mathrm{SU}(3)$ predictions. In other words, the mixing, while strong, appears to be coherent. Chairul Bahri (Draayer's former student) and David Rowe term this 'quasi-dynamical symmetry'28 and relate it to 'adiabatic decoupling of colective motion along the lines of the Born-Oppenheimer approximation.' They considered the symplectic shell model with the Davidson interaction, and found when the wavefunctions are fragmented over many SU(3) irreps, the spectra still look remarkably like $\mathrm{SU}(3)$ rotors. So even when microscopic wavefunctions are not good $\mathrm{SU}(3)$ states, the spectral properties still look like $\mathrm{SU}(3)$.

There are several lessons to take away from this. First, that $\mathrm{SU}(3)$ irreps are not intrinsically very good microscopic wavefunctions-and spinorbit splitting is mainly responsible. Second, and paradoxically, SU(3)-like behavior is very robust, suggesting that one might be able to coherently mix $\mathrm{SU}(3)$ wavefunction to get both the microscopic description and the phenomenology correct. This, I believe, is critical for application to largescale, $a b$ inito shell-model calculations.

The final lesson is that collective behavior does not appear very sensitive to the details of the Hamiltonian. This leads me to my next topic.

\subsection{Collectivity and random interactions}

The above results argue that collective behavior is robust even when one adds 'messy' or 'noisy' pieces to an algebraic Hamiltonian. One can take this to the extreme and ask: if one leaves off the algebraic Hamiltonian altogether and just have a 'noisy' or random Hamiltonian, does any collective behavior remain?

Surprisingly, the answer is yes. If one has a random two-body interaction in a fermion shell model, one sees robust signatures of pairing collectivity ${ }^{29}$. If one has a random two-body interaction in the interacting boson model, one sees robust rotational and vibrational band structures ${ }^{30}$. (See also Roelof Bijker's contribution.) To date, however, no one has found a convincing random ensemble for the fermion shell model that gives rise to robust band structure, despite some proposals ${ }^{31}$. 
There have been a large number of papers written analyzing and purporting to explain the pairing-like behavior in fermion models, but to my mind none of them are terribly convincing. We still need a general theory of how collective behavior can arise generically can arise, a more general version of Bahri and Rowe's quasi-dynamical symmetry. The situation reminds me of quantum chaos: the quantum wavefunctions of classically chaotic systems display 'scars' of classical periodic, but unstable, orbits.

\section{Conclusion}

I lay out two challenges for the intersection of algebraic models with largebasis shell-model diagonalization:

First, one must account for both deformation and spin-orbit splitting. If $\mathrm{SU}(3)$-symplectic wavefunctions are to be an efficient computational basis for large-scale $a b$ initio calculations, we must generalize further to account for spin-orbit mixing a priori.

Second, we need to investigate further how collective behavior arises generically and how it remains robust even in the presence of messy interactions. A good explanation might help answer the first challenge.

\section{Acknowledgements}

It was a pleasure and an honor to be invited to give this overview talk at the celebration of Jerry Draayer's 60th birthday. The bibliography below gives just a taste of his many contributions to nuclear structure theory.

The work described herein was funded by the U.S. Department of Energy and the National Science Foundation.

\section{References}

1. H. Kamada et al., Phys. Rev. C 64, 044001 (2001); R. B. Wiringa, S. C. Pieper, J. Carlson, and V. R. Pandharipande, Phys. Rev. C 62, 014001 (2001); P. Navratil, J.P. Vary, and B.R. Barrett, Phys. Rev. Lett. 84, 5728 (2000); Phys. Rev. C 62, 054311 (2000).

2. I. Talmi, Simple Models of Complex Nuclei (Harwood Academic Publishers, Chur, Switzerland, 1993).

3. D. J. Rowe, Prog. Part. Nucl. Phys. 37, 268 (1996).

4. M. G. Mayer, Phys. Rev. 75, 1969 (1949); O. Haxel, J. H. D. Jensen, and H. E. Suess, Phys. Rev. 75, 1766 (1949).

5. K. T. Hecht and A. Adler, Nucl. Phys. A137, 129 (1969); A. Arima, M. Harvey, and K. Shimizu, Phys. Lett. 30B, 517 (1969).

6. C. Bahri, J. P. Draayer, and S. A. Moszkowski, Phys. Rev. Lett. 68, 2133 (1992); J. N. Ginocchio, Phys. Rev. Lett. 78, 436 (1997). 
7. A. Bohr and B. R. Mottelson, Nuclear Structure, Vol II (W. A. Benjamin, Inc., Boston, 1975).

8. J. P. Elliott, Proc. Roy. Soc. (London) A245, 128 and 562 (1958); M. Harvey, Adv. Nucl. Phys. 1, 67 (1968).

9. R. R. Whitehead, et al, Adv. Nucl. Phys. 9, 123 (1977).

10. A. Etchegoyen, et al, MSU-NSCL Report 524 (1985).

11. E. Caurier, computer code ANTOINE, CRN, Strasbourg, 1989 (unpublished); E. Caurier, A. P. Zuker, and A. Poves, in Nuclear Structure of Light Nuclei Far from Stability, Proceedings of the Obernai Workshop, 1989, edited by G. Klotz (CRN, Strasbourg, 1989).

12. W. E. Ormand, private communication.

13. J. P. Draayer and Y. Akiymka, J. Math. Phys. 14, 1904 (1973); Y. Akiyama and J. P. Draayer, Comp. Phys. Comm. 5, 405 (1973).

14. C. Bahri and J. P. Draayer, Comp. Phys. Comm. 83, 59(1994).

15. J. P. Draayer and K. J. Weeks, Ann. of Phys. 156, 41 (1984); O. Castaños, J. P. Draayer and Y. Lebscher, Ann. of Phys. 180, 290 (1987).

16. C. Bahri, J. Escher, and J. P. Draayer, Nucl. Phys. A 592, 171 (1995).

17. J. Escher, C. Bahri, D. Troltenier, and J. P. Draayer, Nucl. Phys. A 633, 662 (1998).

18. G. Rosensteel and D. J. Rowe, Phys. Rev. Lett. 38, 10 (1977); Ann. Phys. (N.Y.) 126, 343 (1980).

19. J. P. Draayer, K.J. Weeks and G. Rosensteel, Nucl. Phys. A413, 215 (1987); J. Escher and J. P. Draayer, Phys. Rev. Lett. 82, 5221 (1999).

20. V. G. Gueorguiev, J. P. Draayer, and C. W. Johnson, Phys. Rev. C 63, $014318(2000)$.

21. C. W. Johnson, I. Stetcu, and J. P. Draayer, Phys. Rev. C 66, 034312 (2002).

22. B.H. Wildenthal, Prog. Part. Nucl. Phys. 11, 5 (1984).

23. T.T.S. Kuo and G.E. Brown, Nucl. Phys. A114, 235 (1968); A. Poves and A.P. Zuker, Phys. Rep. 70, 235 (1981).

24. E. Caurier, A. Poves, and A. P. Zuker, Phys. Lett. B 252, 13 (1990); Phys. Rev. Lett. 74, 1517 (1995).

25. V. Gueorguiev, W. E. Ormand, C. W. Johnson, and J. P. Draayer, Phys. Rev. C 65, 024314 (2002).

26. T. Hjelt, K. W. Schmid, and A. Faessler, Nucl. Phys. A 697, 164 (2002); E. Bender, K. W. Schmid and A. Faessler, Prog. Part. Nucl. Phys. 38, 159 (1997); V. Velázquez, J. G. Hirsch and Y. Sun, Nucl. Phys. A 643, 39 (1998); R. Sahu and V. K. B. Kota, Phys. Rev. C 66, 024301 (2002).

27. M. J. Carvalho, D. J. Rowe, S. Karram, and C. Bahri, Nucl. Phys. A 703, 167 (2002).

28. C. Bahri and D. J. Rowe, Nucl. Phys. A 662, 125 (2000).

29. C. W. Johnson, G. F. Bertsch, and D.J.Dean, Phys. Rev. Lett. 80, 2749 (1998); C. W. Johnson, G. F. Bertsch, D. J. Dean, and I. Talmi, Phys. Rev. C 61, 014311 (2000).

30. R. Bijker and A. Frank, Phys. Rev. Lett. 84, 420 (2000).

31. V. Velázquez and A. P. Zuker Phys. Rev. Lett. 88, 072502 (2002). 